\title{
Effect of Plastic Strain Range on Prediction of the Onset of Crack Growth for Low-Cycle Fatigue of SUS316NG Studied using Ultrasonic Back-Reflection*
}

\author{
Islam Md. NURUL ${ }^{* *}$, Yoshio ARAI ${ }^{* *}$ and Wakako ARAKI ${ }^{* *}$ \\ ${ }^{* *}$ Graduate School of Science and Engineering, Saitama University \\ 255 Shimo-ohkubo, Sakura-ku, Saitama 338-8570, Japan \\ E-mail:yarai@mech.saitama-u.ac.jp
}

\begin{abstract}
Strain range controlled low-cycle fatigue tests were conducted using ultrasonic method in order to investigate the effect of plastic strain range on the remaining life of austenitic stainless steel SUS316NG before the onset of crack growth in its early stages of fatigue. It was found that the decrease in ultrasonic back-reflection intensity from the surface of the material, caused by the increase in average dislocation density with localized plastic deformation at persistent slip bands (PSBs), starts earlier with increase in the plastic strain range. The amount of decrease in ultrasonic back-reflection before the onset of crack growth increases for larger plastic strain range. The difference in the cumulative plastic strains at the onset of crack growth and at the onset of decrease in the ultrasonic back-reflection remained constant over the range of tested plastic strain. This result can be used to predict the remaining life before the onset of crack growth within the plastic strain range used in this study. In addition, we present and evaluate another method to predict damage evolution involving ultrasound attenuation caused by PSBs.
\end{abstract}

Key words: Plastic Strain Range, Ultrasonic Back-reflection, Austenitic Stainless Steel, Low Cycle Fatigue, Crack Growth

\section{Introduction}

It is well established that in low-cycle fatigue, emergence of slip bands and crack initiation occurs at the free surface of metal and metallic alloy components subjected to fatigue loading ${ }^{(1-5)}$. Cyclic plastic strain range plays an important role in the localization of cyclic plastic straining and the crack initiation. The localization of cyclic plastic strain begins at very early stages of fatigue life, forming persistent slip bands (PSBs) ${ }^{(6,7)}$. In polycrystalline nickel, the volume fraction of PSBs within a grain and the volume fraction of grains occupied by PSBs, increase with increasing plastic strain range ${ }^{(8)}$. As a result of repeated loading, the PSB density saturates and localized cyclic plastic straining in PSBs is observed, followed by crack initiation along the PSBs ${ }^{(9,10)}$. Furthermore, onset of crack growth occurs from the initiated crack along the PSBs without affecting other regions. In such a case, knowledge of the local fatigue damage plays an important role in predicting the remaining fatigue life.

Because of its easy and simple procedure of implementation, ultrasonic method has been extensively used as a technique to monitor progressive changes in material properties. For example, the change in attenuation coefficients with continuing fatigue for carbon steel, 
polycrystalline copper, and aluminum alloys have been reported ${ }^{(11-15)}$. These studies performed through-thickness measurements of the average attenuation value using a low frequency ultrasound transducer. In our previous research, a method using ultrasonic back-reflection to detect the onset of low-cycle fatigue crack growth from cracks initiated along PSBs under a constant load amplitude was proposed ${ }^{(16)}$. To evaluate the low-cycle fatigue life using the proposed ultrasonic back-reflection method the influence of the load magnitude (plastic strain range) on the results of the method should be examined. In the current study, the dependence of ultrasonic back-reflection on the plastic strain range at the location where low-cycle fatigue crack growth starts in stainless steel was evaluated and methods to predict the remaining life in terms of the onset of crack growth for a range of plastic strain amplitudes were proposed.

\section{Materials and Methods}

The material investigated in this experiment was an austenite stainless steel (JIS-SUS316NG) ${ }^{(17)}$. Its chemical compositions and mechanical properties are given in Tables 1 and 2, respectively. The specimen shape, dimensions, and the polishing procedure were described previously ${ }^{(16)}$. The microstructure is shown in Fig. 1, and the average grain diameter is $100 \mu \mathrm{m}$.

Table 1 Chemical compositions [wt.\%]

\begin{tabular}{|c|c|c|c|c|c|c|c|c|c|c|c|c|c|}
\hline $\mathrm{Cr}$ & $\mathrm{Ni}$ & $\mathrm{C}$ & $\mathrm{N}$ & $\mathrm{Mn}$ & $\mathrm{Si}$ & $\mathrm{S}$ & $\mathrm{P}$ & $\mathrm{Mo}$ & $\mathrm{Cu}$ & $\mathrm{B}$ & $\mathrm{Co}$ & $\mathrm{As}$ & $\mathrm{Fe}$ \\
\hline 17.4 & 11.9 & 0.02 & 0.07 & 1.69 & 0.31 & 0.002 & 0.023 & 2.25 & 0.11 & 0.009 & 0.19 & 0.004 & $\mathrm{Bal}$ \\
\hline
\end{tabular}

Table 2 Mechanical properties

\begin{tabular}{|c|c|c|c|}
\hline $\mathrm{E}(\mathrm{GPa})$ & $v$ & $\sigma_{0.2}(\mathrm{MPa})$ & $\sigma_{\mathrm{B}}(\mathrm{MPa})$ \\
\hline 190 & 0.25 & 261 & 583 \\
\hline
\end{tabular}



Fig. 1 Micro-structure of tested material

We performed strain-controlled fatigue testing using an electro-hydraulic material testing machine (MTS810), and applied plane-bend loading. The loading cycle is shown in Fig. 2. We investigate three different plastic strain range $\left(\Delta \varepsilon_{p}\right)$ at the specimen surface, $\Delta \varepsilon_{p}=1200 \times 10^{-6}, 1900 \times 10^{-6}$, and $2400 \times 10^{-6}$ in each saturated state (which we 
refer to as low, medium, and high, respectively). $\Delta \varepsilon_{p}$ is defined as $\varepsilon_{\mathrm{p} \text {,max }}-\varepsilon_{\mathrm{p} \text {,min }}$, or the difference between the maximum and minimum plastic strain. The stress ratio was -1 , the loading frequency was $1 \mathrm{~Hz}$, and the fatigue tests were conducted in air. After applying the cyclic loads, test specimen was taken out from the material testing machine and then the surface was observed by an optical microscope (magnification of 1000) in air and a scanning acoustic microscope (HSAM220, HITACHI) in water. High frequency transducer $(100 \mathrm{MHz})$ was used for the evaluation of ultrasonic wave.

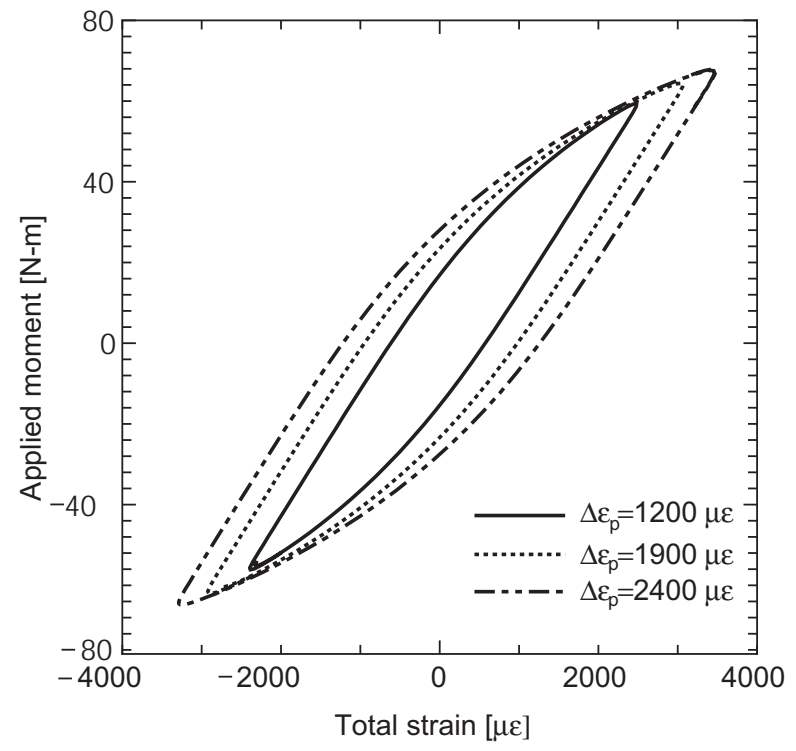

Fig. 2 Three different loading curves

To determine the area fraction of PSBs, $D_{\mathrm{PSB}}$, for each plastic strain range, we measured the length and width of slip bands optically over the same area measured ultrasonically on a test piece surface. Dividing the PSB area by the entire observed area (about $1000 \times$ $1000 \mu \mathrm{m}^{2}$ ) gives $D_{\mathrm{PSB}}$.

Figure 3 shows the ultrasonic wave propagation path. The transducer receives the ultrasonic back-reflected wave from the reflection off the crystal grain boundary. The maximum intensity $A_{\max }$ of the back-reflected wave is normalized by the reference value $A_{0}$ and the ratio $A_{\max } / A_{0}$ is used to monitor changes in back-reflection intensity, which is attributed to fatigue. Surface observations and the detection principle have already been studied ${ }^{(16)}$.

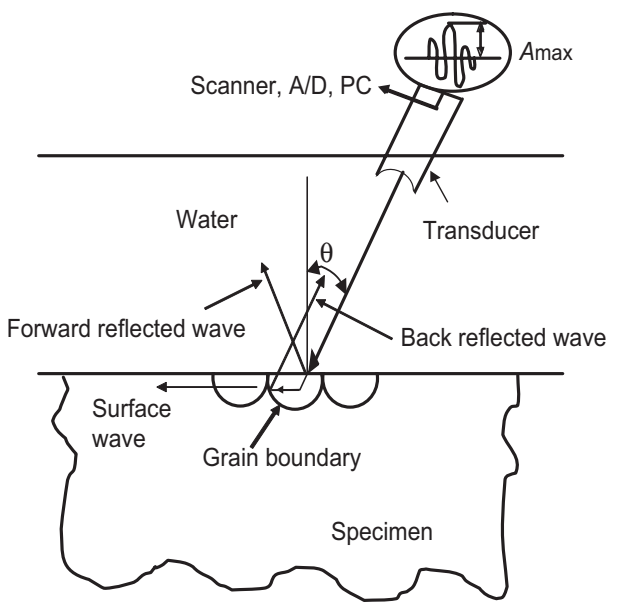

Fig. 3 Ultrasonic wave propagation path 


\section{Experimental Results}

Figure 4 shows the evolution of slip bands from the early stage of fatigue life to the onset of crack growth due to different plastic strains range. The slip bands, which appear as black lines in the optical microscope images, increase in number with increasing plastic strain range. Figure 5 shows the relationship between the area fraction occupied by PSBs, $D_{\mathrm{PSB}}$, and $N / N_{\mathrm{f}}\left(N_{\mathrm{f}}\right.$ is the number of load reversals to have failed), for the low, medium, and high plastic strain ranges, respectively. The area fraction occupied by PSBs increase with increasing plastic strain range and also with increasing number of fatigue cycles, and tends to saturate at $N / N_{\mathrm{f}}=0.15, N / N_{\mathrm{f}}=0.14$, and $N / N_{\mathrm{f}}=0.12$ for low, medium, and high strain ranges, respectively. We estimated the saturation as the change in $D_{\mathrm{PSB}}$ less than $0.5 \%$.
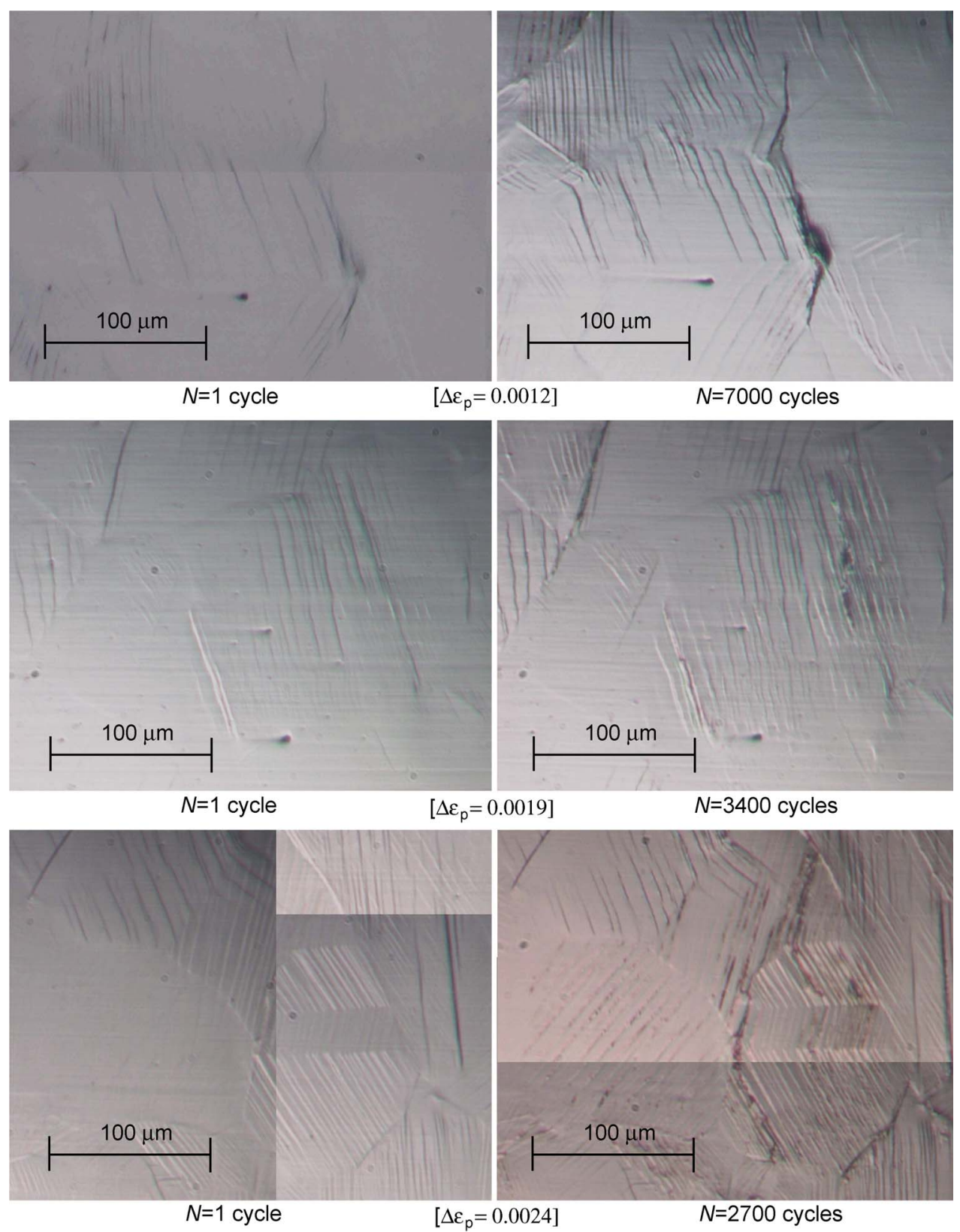

Fig. 4 Comparison of slip band evolution for different plastic strain range. $N=1$ and $N=$ $7000, N / N_{\mathrm{f}}=0.125$ for $\Delta \varepsilon_{\mathrm{p}}=0.0012, \quad N=3400, N / N_{\mathrm{f}}=0.13$ for $\Delta \varepsilon_{\mathrm{p}}=0.0019, N=$ $2700, N / N_{\mathrm{f}}=0.14$ for $\Delta \varepsilon_{\mathrm{p}}=0.0024, N_{\mathrm{f}}$ are 56000,27300 and 19400 for different plastic range, respectively. 


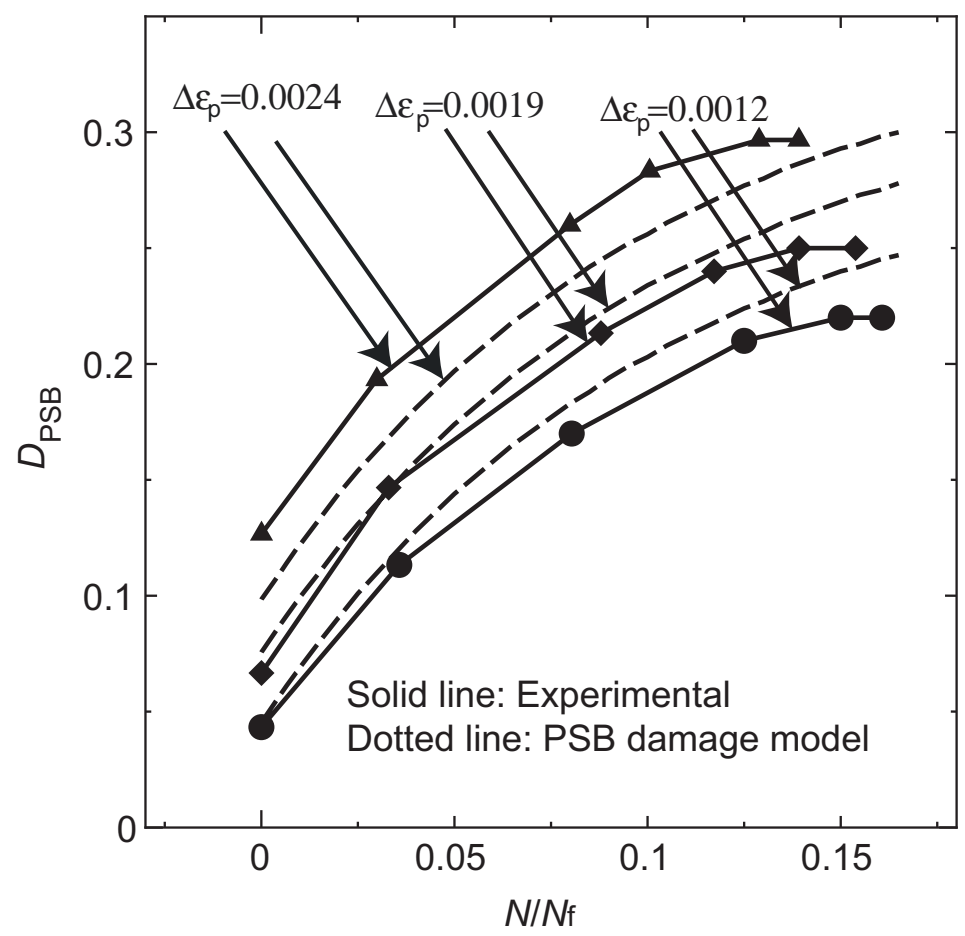

Fig.5 Relationship between area fraction occupied by PSBs, $D_{\mathrm{PSB}}$ and fraction of fatigue life

Figure 6 shows ultrasonic microscope images for low, medium, and high plastic strain ranges from an early stage of fatigue to the onset of crack growth. In the ultrasonic microscope image, the inhomogeneous brightness distribution indicates the back-reflection intensity from grain boundaries. The rectangular mark indicates the location where crack growth begins. The brightness of the ultrasonic image in this location decreases at $N=4000$ compared to the image at $N=1$ because of fatigue damage accumulation (left side image). The brightness starts to increase at $N=10,200$ for the low plastic strain range. Similarly, for the medium plastic strain range, the brightness of the ultrasonic image starts to decrease from $N=4600$ and then starts to increase with increasing load cycle at $N=5300$ (middle column image). At high plastic strain range, the brightness of ultrasonic image decreases at $N=700$ and then starts to increase at $N=1350$. The large decrease affects the brightness of the back-reflected image for medium and high strain range, whereas the decrease in brightness for low strain range is relatively small. Image representation of change in back reflection intensity will be useful for the fatigue damage detection and is described in detail in Appendix A. As shown in Fig. 7, optical microscope observations of the same location and for the corresponding number of cycles show that the length of the black line (slip band or crack) remains constant during the period before the brightness of the rectangular area in Fig. 6 increases. The black line begins increasing in length at the same time that the brightness of the rectangular area starts to increase, indicating the start of crack growth from a crack initiated along a PSB.

Figure 8 shows typical examples of the dependence of ultrasonic back-reflection intensity on fatigue loads, with the three plastic strain ranges. The solid symbols represent the reflected wave intensity from the grain boundaries, including PSBs where crack growth is observed, and open symbols represent the change in intensity from the grain boundaries including PSBs subjected to the same cyclic deformation, but where no crack growth is observed. In this figure the data for the low plastic strain range and the data for no crack growth are assigned the same origin, $A_{\max } / A_{0}$. For PSBs at medium and high plastic strain range, the origins are shifted to clarify the illustration. The upper arrow mark indicates $N / N_{\mathrm{f}}$ at the start of crack growth for different strain ranges. Figure 8 shows that the number of 




$N=1, \quad \Delta \varepsilon_{p}=0.0012$


$N=8400, \Delta \varepsilon_{p}=0.0012$



$N=10200, \Delta \varepsilon_{p}=0.0012$

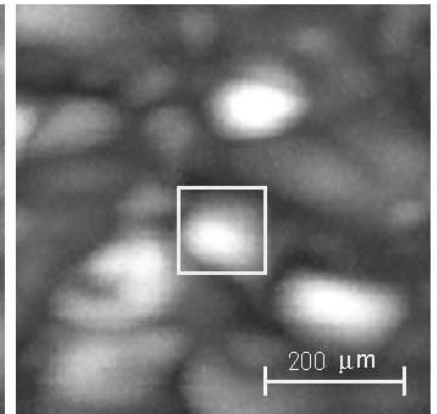

$N=1$

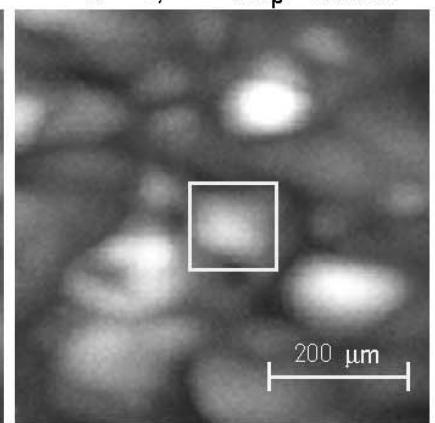

$N=4600, \quad \Delta \varepsilon_{p}=0.0019$



$N=5000, \Delta \varepsilon_{p}=0.0019$

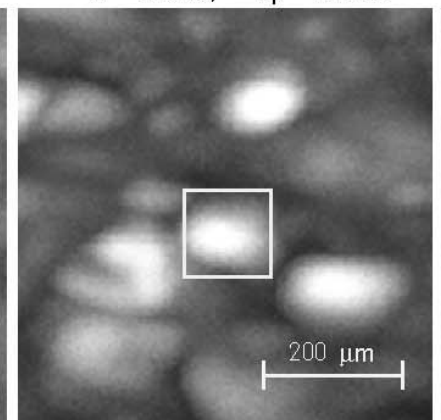

$N=5300, \Delta \varepsilon_{p}=0.0019$



$N=1, \quad \Delta \varepsilon_{p}=0.0024$

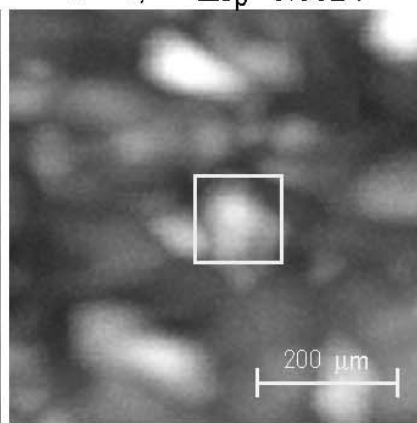

$N=700, \quad \Delta \varepsilon_{p}=0.0024$

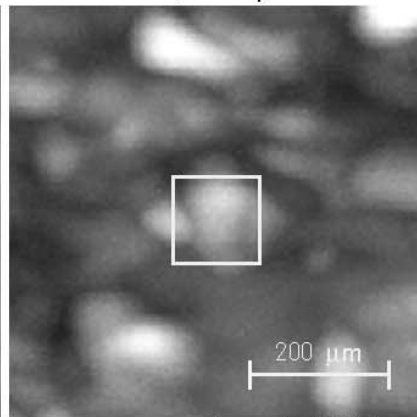

$N=900, \quad \Delta \varepsilon_{p}=0.0024$

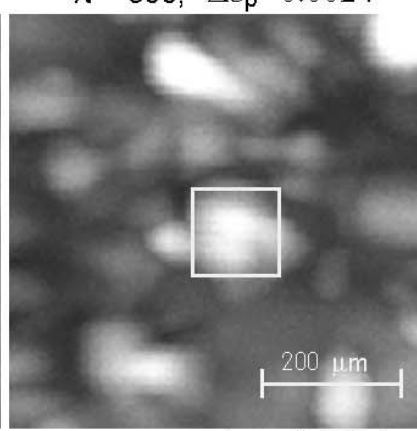

$N=1350, \quad \Delta \varepsilon_{p}=0.0024$

Fig.6 Comparison of ultrasonic microscope images for three different strain ranges

cyclic loadings before the onset of decrease in the ultrasonic back-reflection and the onset of crack growth are decreased with increasing plastic strain range. For low strain range, the ultrasonic back-reflection shows approximately $30 \%$ decrease in intensity from the initial value to the minimum when the crack growth starts, whereas for medium and high strain ranges, the decrease is of about $40 \%$ and $44 \%$, respectively. The start of decrease in ultrasonics which is leading to the start of crack growth occurred about $1 \sim 11 \%$ of fatigue life. The number of cycles when the decrease in ultrasonics starts is defined as $5 \%$ decrease compared with the average value of no crack initiated location within the range. 


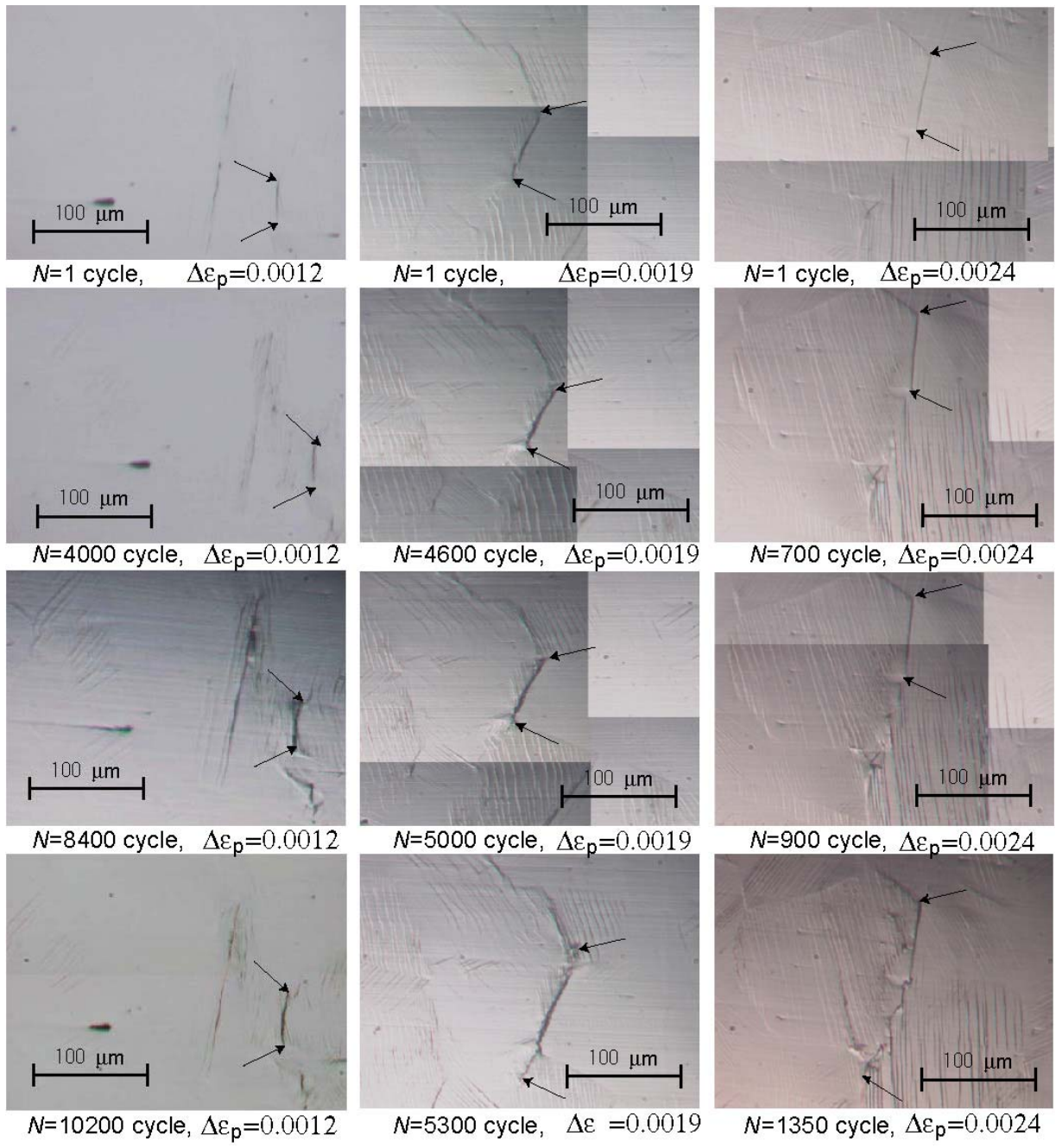

Fig.7 Optical microscope images corresponding to Fig. 6

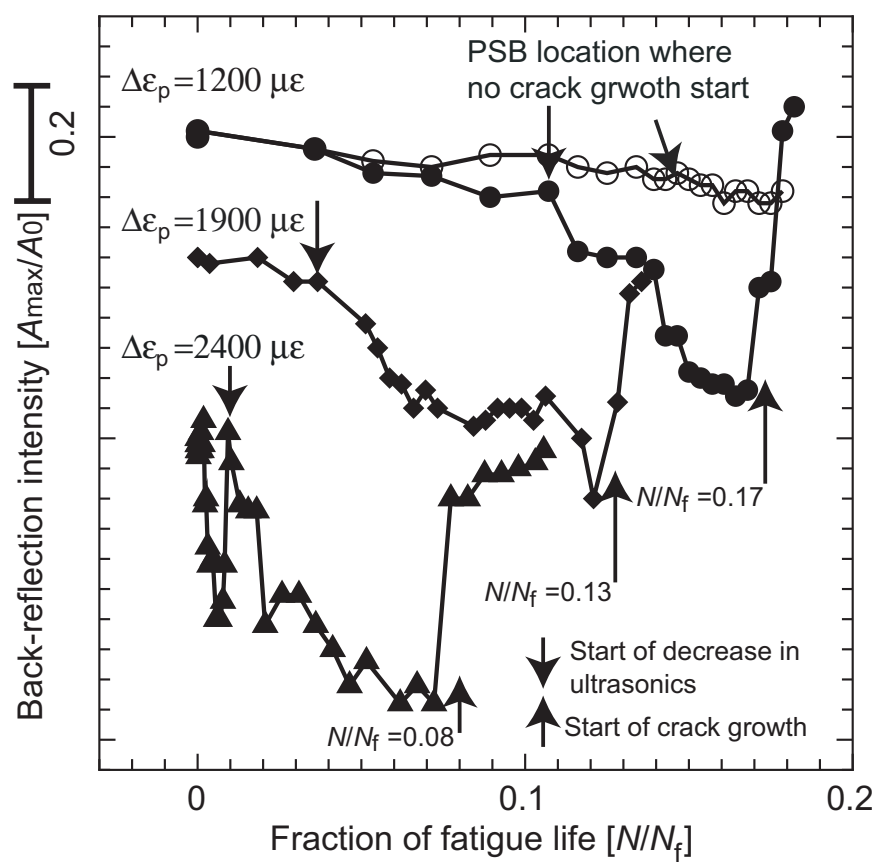

Fig. 8 Typical example of relationship between back reflection intensity, $A_{\max } / A_{0}$ and number of fatigue 
The number of cycles $N_{\mathrm{su}} / N_{\mathrm{f}}$ (SU stands for start of decrease in ultrasonics and $N_{\mathrm{su}}$ is the corresponding number of cycles) at the start of decrease in the ultrasonic back-reflection is shown as a function of plastic strain range in Fig. 9. The boundary $N_{\mathrm{sL}} / N_{\mathrm{f}}$ (SL stands for strain localization and $N_{\mathrm{sL}}$ is the corresponding number of cycles) in copper ${ }^{(18)}$ between the regime of cyclic plastic strain localization (designated as regime II in Ref. (18)) and the regime of repeated cyclic plastic strain localization at PSBs from where cracks are nucleated (regime III) is also plotted versus plastic strain range in Fig. 9. The two quantities $N_{\mathrm{su}} / N_{\mathrm{f}}$ and $N_{\mathrm{sL}} / N_{\mathrm{f}}$ have a similar dependence on plastic strain range. The onset of decrease

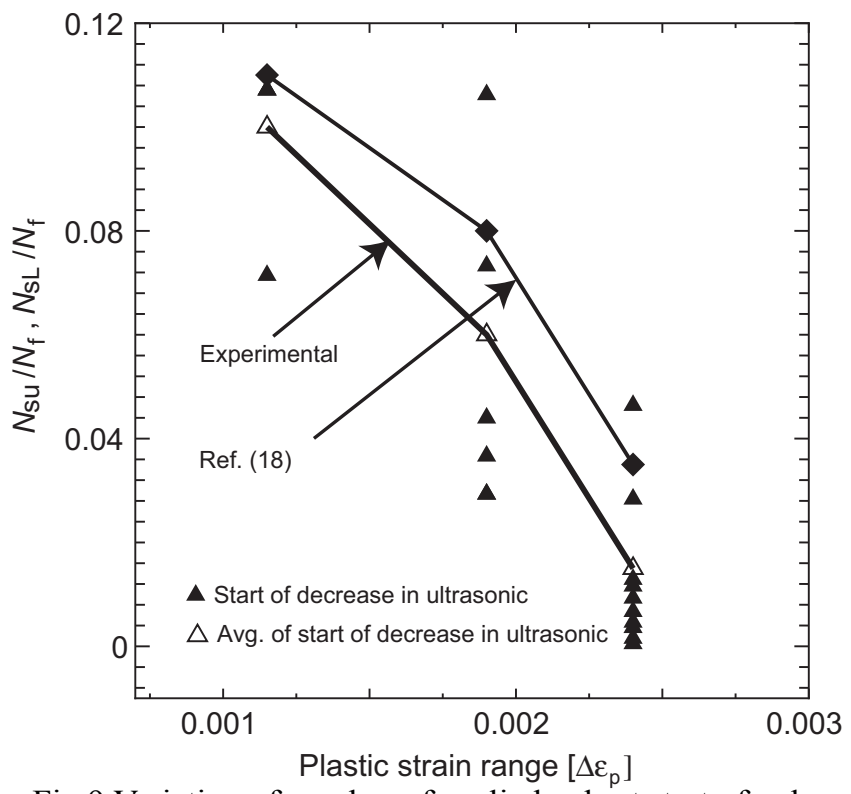

Fig.9 Variation of number of cyclic loads at start of decrease in ultrasonics with plastic strain range

in the ultrasonic back-reflection occurs near the end of regime II (development of PSBs) and the beginning of regime III (saturation of PSB volume fraction and initiation of microscale cracks). The high plastic strain range leads to early saturation of the PSB volume fraction and the localization of repeated cyclic plastic straining in PSBs. The amount of decrease in ultrasonic back-reflection versus plastic strain range is shown in Fig. 10. The larger decrease in the ultrasonic back-reflection mirrors the evolution of the damage, or the area fraction occupied by PSBs and the dislocation density in PSBs.

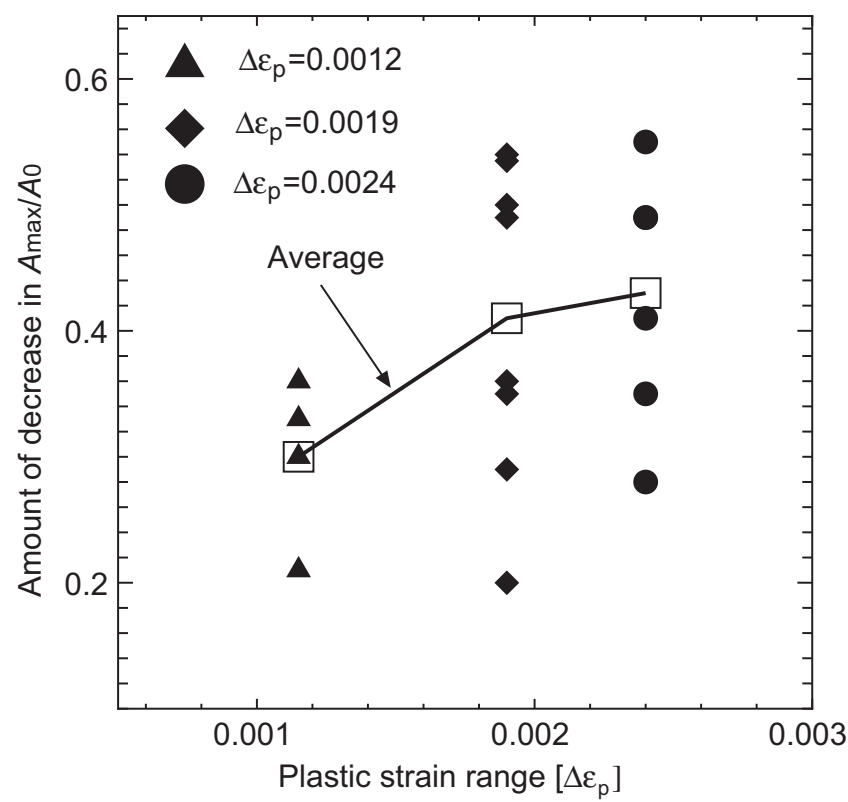

Fig.10 Comparison on amount of decrease in $A_{\max } / A_{0}$ 
Figure 11 shows that the cumulative plastic strain $2 \Delta \varepsilon_{p} N$ at the onset of decrease in the ultrasonic back-reflection $N_{\mathrm{su}}$ and at the start of crack growth $N_{\mathrm{sc}}$ (SC stands for start of crack growth and $N_{\mathrm{sc}}$ is the corresponding number of cycles) decreases with increasing plastic strain range, whereas the cumulative plastic strain difference at the onset of crack growth and at the onset of decrease in the ultrasonic back-reflection (square symbols) remains almost constant.

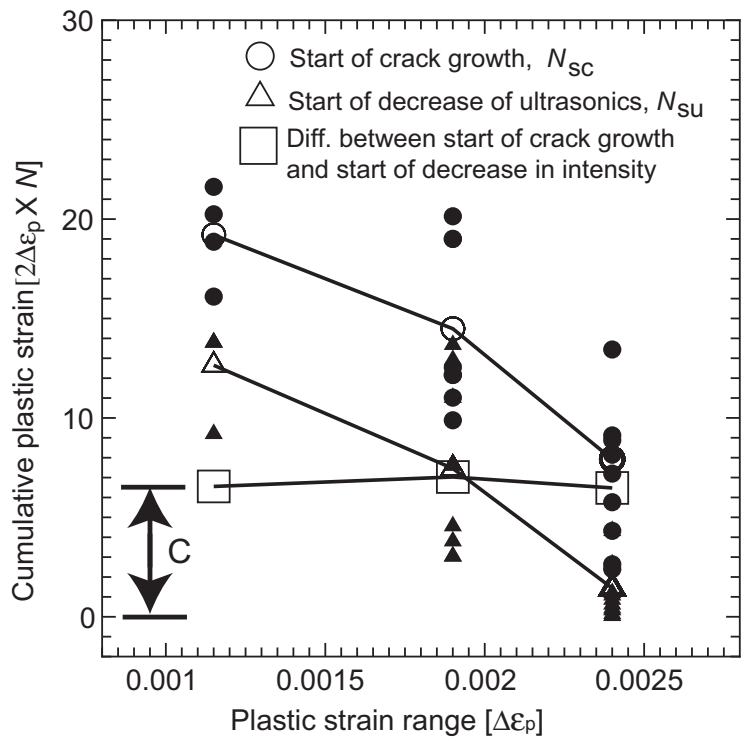

Fig. 11 Relationship between plastic strain range and cumulative plastic strain

\section{Discussions}

The number of cycles before the onset of crack growth can be predicted using

$$
N_{\mathrm{sc}}=N_{\mathrm{su}}+\Delta N_{\mathrm{sc}}
$$

where $N_{\mathrm{su}}$ can be measured using the ultrasonic method described above and $\Delta N_{\mathrm{sc}}$ can be calculated using one of the following two methods.

In the first method, we assume a model of damage evolution in a crystal grain that includes PSBs to investigate how the ultrasonic back-reflection decay depends on plastic strain. Figure 5 shows that $D_{\mathrm{PSB}}$ is dependent on plastic strain range, $\Delta \varepsilon_{\mathrm{p}}$, and also on the number of fatigue cycles, $N / N_{\mathrm{f}}$, so we assume the following relation ${ }^{(18)}$ for $N>0$ :

$$
D_{\mathrm{PSB}}=\bar{D}_{\mathrm{PSB}}\left[\left\{1-\exp \left(-\kappa_{\varepsilon} \Delta \varepsilon_{\mathrm{p}}\right)\right\}+\left\{1-\exp \left(-\kappa_{\mathrm{N}} \cdot \frac{N}{N_{\mathrm{f}}}\right)\right\}\right],
$$

where $\bar{D}_{\mathrm{PSB}}$ is half the saturated value of $D_{\mathrm{PSB}}$ when both $\Delta \varepsilon_{\mathrm{p}}$ and $N / N_{\mathrm{f}}$ are large, $\kappa_{\varepsilon}$ is a coefficient for giving the dependence of $D_{\mathrm{PSB}}$ on the plastic strain range, and $\kappa_{\mathrm{N}}$ is a coefficient giving the dependence of $D_{\mathrm{PSB}}$ on the number of cycles. Using $\bar{D}_{\mathrm{PSB}}, \kappa_{\varepsilon}$, and $\kappa_{\mathrm{N}}$ as parameters, we fit Eq. (2) to the experimentally measured relation between $D_{\mathrm{PSB}}$ and $N / N_{\mathrm{f}} \quad$ (see Fig. 5, dotted lines), and find $\bar{D}_{\mathrm{PSB}}=0.25, \kappa_{\varepsilon}=200$, and $\kappa_{\mathrm{N}}=10$. The average dislocation density in the crystal grain including PSBs becomes

$$
\Lambda_{\mathrm{av}}=\Lambda_{\mathrm{PSB}} \times D_{\mathrm{PSB}}+\Lambda_{0}
$$

where $\Lambda_{\text {PSB }}$ is the density of movable dislocations in PSBs and $\Lambda_{0}$ is the initial dislocation density of the grain. Using the value of $\Lambda_{\mathrm{av}}$ from Eq.(3), we estimate the ultrasonic attenuation of the grain including PSBs and the ultrasonic back-reflection intensity from the grain using ${ }^{(19)}$

$$
\alpha=\mathrm{C}_{1} \Lambda_{\mathrm{av}} L^{4} f^{2}
$$




$$
\frac{A_{\max }}{A_{0}}=\exp \left\{-2\left(\alpha_{1}-\alpha_{0}\right) H\right\},
$$

where $\mathrm{C}_{1}$ is the material constant, $L$ is the dislocation loop length, $f$ is the frequency of the ultrasonic wave, $\alpha_{0}$ and $\alpha_{1}$ are coefficients of attenuation for the initial and current states, respectively, and $H$ is the diameter of crystal grain ${ }^{(16)}$. To calculate the attenuation using Eq. (4), we assume $L=160 \mathrm{~nm}{ }^{(20-22)}, \bar{\Lambda}_{\mathrm{PSB}}=6.0 \times 10^{15} \mathrm{~m}^{-2}{ }^{(23)}$, and $\Lambda_{0}=1.0 \times 10^{12} \mathrm{~m}^{-2}{ }^{(20)}$. The critical value of $A_{\max } / A_{0}$ which indicates when the crack growth starts is evaluated from the average amount of decrease in $A_{\max } / A_{0}$ over several PSBs from where the crack growth starts in the experiment. $\Delta N_{\mathrm{sc}} / N_{\mathrm{f}}$ is the increment in $N / N_{\mathrm{f}}$ [see Eq. (2)] when $A_{\max } / A_{0}$ reaches its critical value.

The second method to predict the remaining life before the start of crack growth relies on the cumulative plastic strain. From Fig. 11 it can be seen that the cumulative plastic strain at the onset of crack growth and at the onset of decrease in ultrasonic back-reflection decreases with increasing plastic strain range. Hatanaka ${ }^{(24)}$ established a linear relationship between cumulative plastic strain energy to failure $\left(W_{\mathrm{f}}=\Delta W \times N_{\mathrm{f}}=\Delta \sigma \times \Delta \varepsilon_{\mathrm{p}} \times N_{\mathrm{f}}\right)$ and plastic strain $\left(\Delta \varepsilon_{\mathrm{p}}\right)$ in a study on criteria of low-cycle fatigue fracture in aluminum, Al-2.6\% Mg alloy, copper, $\alpha$-brass, and low carbon steel. This relation indicates that, under constant stress amplitude, fatigue fracture occurs when the cumulative plastic strain reaches a critical value. In analogy to this relation we assume that, under constant stress, the difference in the cumulative plastic strain between the onset of decrease in ultrasonic back-reflection (i.e., the beginning of extensive damage due to repeated plastic straining in PSBs) and at the onset of crack growth (i.e., the end of crack extension in the grain) remains almost constant, which is what we find experimentally (see Fig. 11). The remaining life until the onset of crack growth can be predicted using

$$
\Delta \mathrm{N}_{\mathrm{sc}}=\frac{C}{2 \Delta \varepsilon_{\mathrm{p}}},
$$

where $C$ is a material constant giving the difference in cumulative plastic strain between the decrease in the ultrasonic back-reflection and the onset of crack growth, shown as an arrow in Fig. 11. The simulation results of $A_{\max } / A_{0}$ vs $N / N_{\mathrm{f}}$ using Eqs. (2) (5) compared to the linear approximation method ${ }^{(16)}$ and the predicted start of crack growth using the methods proposed are shown in Figs. 12, 13, and 14 for three different plastic strain ranges respectively. A point from where the simulation was conducted was chosen to be the point at which the large decrease in ultrasonic back-reflection intensity begins. Four different color arrows indicate the start of crack growth as detected experimentally, from the linear approximation, from the PSB damage model using Eqs. (2) (5), and from the constant cumulative plastic strain model Eq. (6). In Fig. 12 we can see that the onset of crack growth given by the PSB damage model is close to the experimental value for three PSB locations out of four. In Fig. 13 too, three out of four PSB locations show the same trend, whereas in Fig. 14 the corresponding values are five out of six. These results indicate that the PSB damage model proposed herein is more effective than the previously proposed method ${ }^{(16)}$. The constant cumulative plastic strain model gives results similar to those of the PSB damage model over the range of plastic strain range tested in this study. After the onset of decrease in the ultrasonic back-reflection, the dominant macroscopic parameter influencing fatigue crack growth start is the cumulative plastic strain.

In this research as we described earlier that after applying cyclic loads, taking out the test specimen and then the surface was observed by optical and ultrasonic microscope. When we applied this technique to low cycle fatigue, the number of off-site observations was reasonable with the small interval of fatigue cycle in order to observe the start of decrease in ultrasonics and also the start of crack growth. If this technique is applied to high cycle fatigue, it will be important to catch the start of decrease in ultrasonics as well as the start of crack growth on-site during fatigue test. 


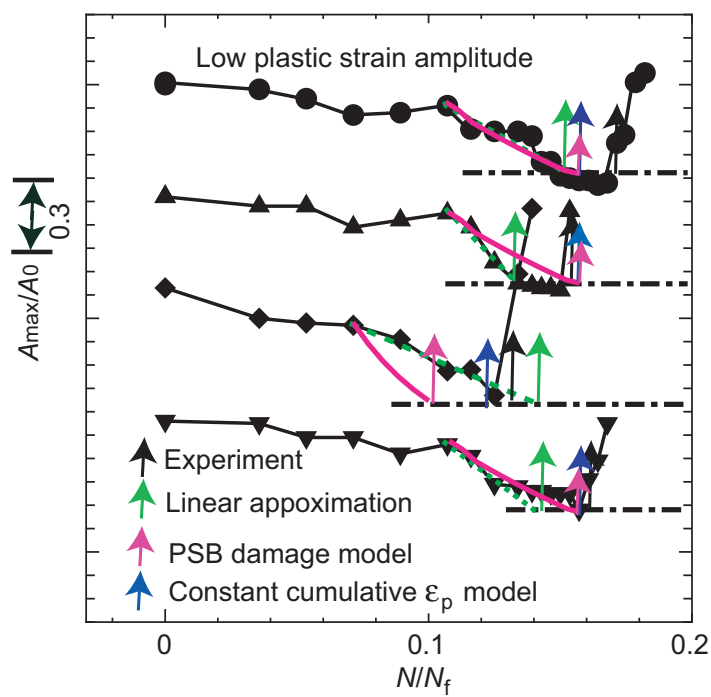

Fig.12 Relationship between back reflection intensity and fraction of fatigue life from simulation, $\Delta \varepsilon_{\mathrm{p}}=0.0012$

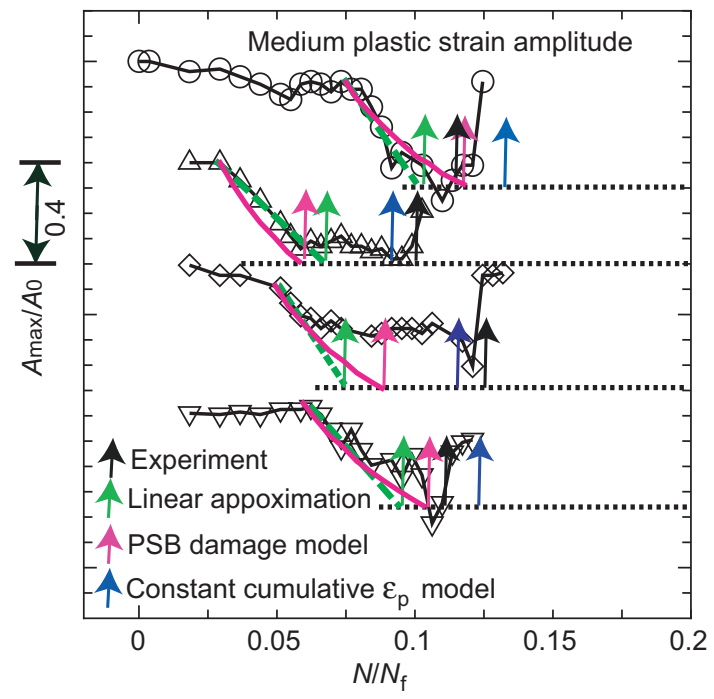

Fig.13 Relationship between back reflection intensity and fraction of fatigue life from simulation, $\Delta \varepsilon_{\mathrm{p}}=0.0019$

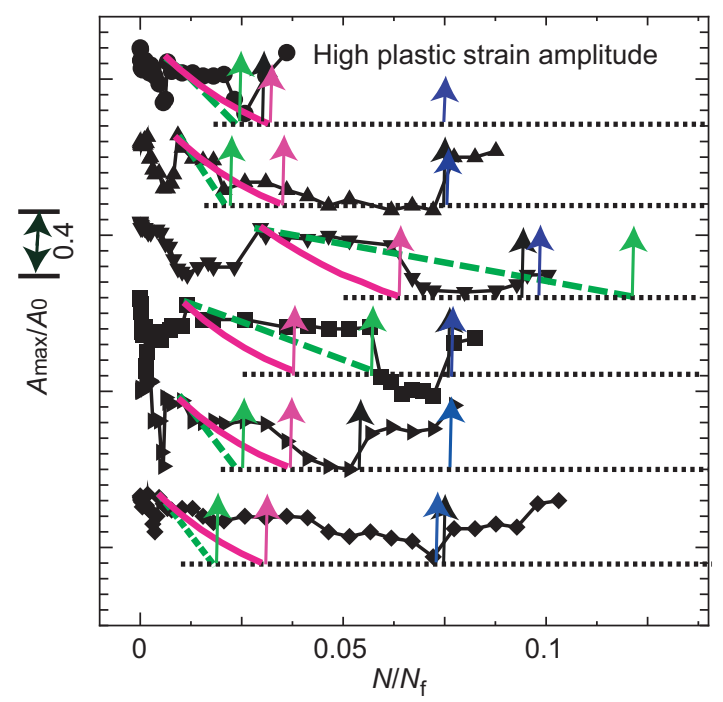

Fig.14 Relationship between back reflection intensity and fraction of fatigue life from simulation, $\Delta \varepsilon_{\mathrm{p}}=0.0024$. Arrows bears the same meaning as indicated in Figs.12 and 13. 


\section{Conclusions}

The results reported herein indicate that ultrasonic back-reflection depends on plastic strain range for low-cycle fatigue of stainless steel SUS316NG. Based on the data presented, we conclude:

1. The number of cycles before the onset of decrease in the ultrasonic back-reflection decreases with increasing plastic strain range. The amount of decrease in the ultrasonic back-reflection before the onset of crack growth increases with increasing plastic strain range; approximately $30 \%$ for low strain, $40 \%$ for medium strain, and $44 \%$ for high strain.

2. The method proposed using PSB damage evolution to be more effective in predicting the remaining life before the onset of crack growth.

3. Differences in cumulative plastic strain between the onset of crack growth and the onset of decrease in the ultrasonic back-reflection remain almost constant for different strain ranges, making it possible to predict the remaining fatigue life.

\section{Acknowledgement}

The authors express gratitude to the Ministry of Education, Science, Sports, and Culture, of the Government of Japan for providing financial support during this research work and also to the LCF committee in The Japan Welding Engineering Society supported by Tokyo Electric Power Company, Hokkaido Electric Power Co., Inc., Tohoku Electric Power Co., Inc., Chubu Electric Power Co., Inc., Hokuriku Electric Power Company, The Kansai Electric Power Co,. Inc., The Chugoku Electric Power Co., Inc., Shikoku Electric Power Co., Inc., Kyusyu Electric Power Co., Inc., The Japan Atomic Power Company and Electric Power Development Co., Ltd.

\section{Appendix A}

\section{Image representation of change in ultrasonic back reflection intensity}

Corresponding to Fig. 6, at first we select corresponding frames in the image of $\mathrm{N}=1$, $\Delta \varepsilon_{\mathrm{p}}=0.0012$ and $\mathrm{N}=8400, \Delta \varepsilon_{\mathrm{p}}=0.0012$. Then we subtract the value of intensity from $\mathrm{N}=1$, $\Delta \varepsilon_{\mathrm{p}}=0.0012$ to $\mathrm{N}=8400, \Delta \varepsilon_{\mathrm{p}}=0.0012$, point by point and we plot the intensity change on the selected location of $2 \mathrm{D}$ plane as a contour in which the red means large intensity change and the blue means small intensity change. The same procedure follows for the results of $\Delta \varepsilon_{\mathrm{p}}=0.0019$ and $\Delta \varepsilon_{\mathrm{p}}=0.0024$. The results are shown in Figs. A1, A2 and A3. From the results we can see that the fatigue damaged area (the rectangular mark region) is highlighted which indicates high quality representation as a fatigue damage detection method. 


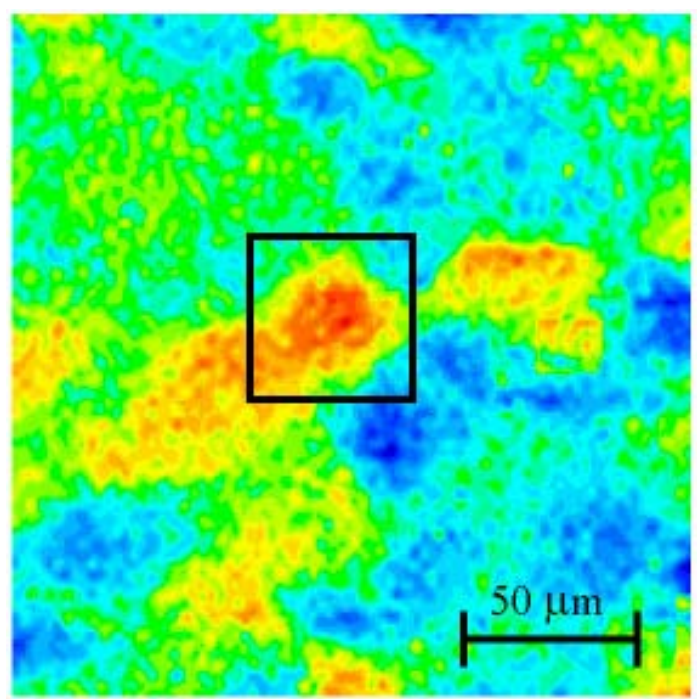

Fig. A1 Difference in intensity from $N=1$ to $N=8400$ $\left(\Delta \varepsilon_{\mathrm{p}}=0.0012\right.$, left column image in Fig. 6)

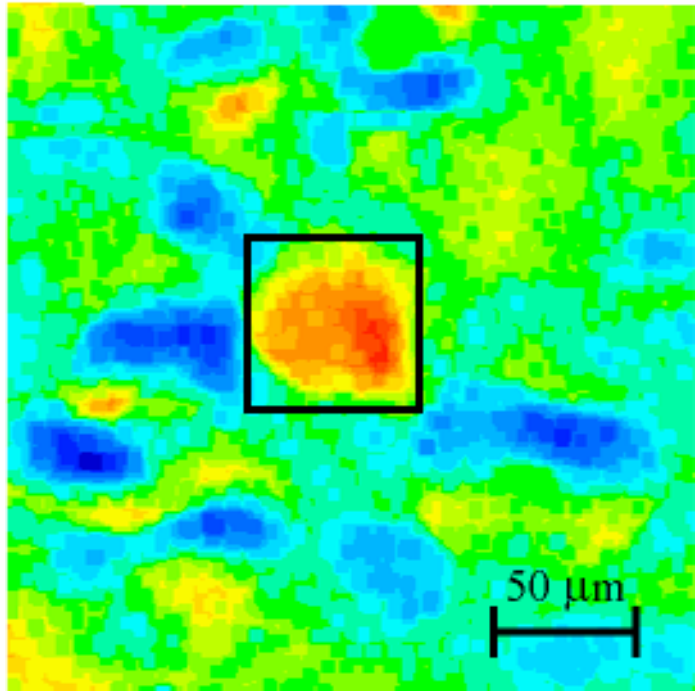

Fig. A2 Difference in intensity from $N=1$ to $N=5000$ $\left(\Delta \varepsilon_{\mathrm{p}}=0.0019\right.$, middle column image in Fig. 6)

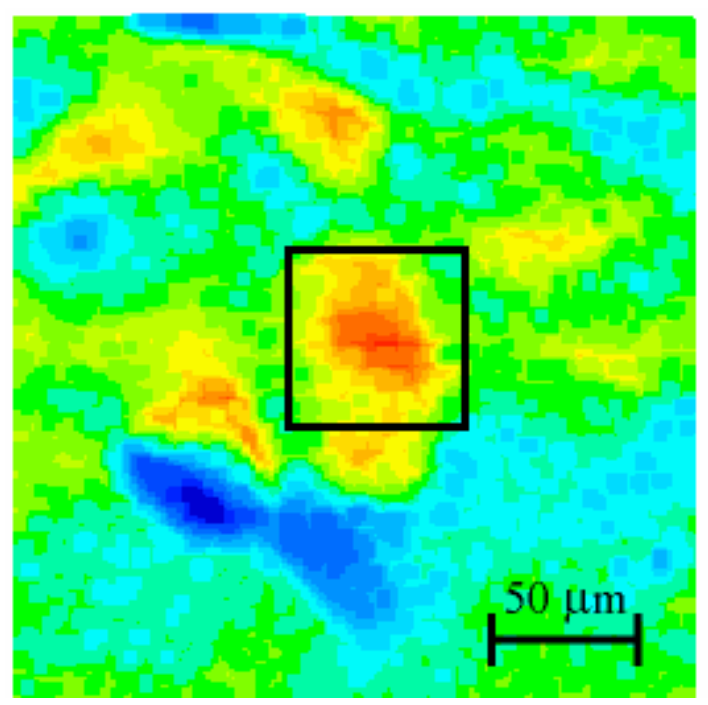

Fig. A3 Difference in intensity from $N=1$ to $N=900$ $\left(\Delta \varepsilon_{\mathrm{p}}=0.0024\right.$, right column image in Fig. 6) 


\section{References}

(1) Ewing, J. A. and Humfrey, J. C., The Fracture of Metals under Repeated Alternations of Stress, Philosophical Transactions of the Royal Society, Vol. A200, (1903), pp. $241-250$.

(2) Baniski, Z.S. and Baniski, S.J., Acta Metallugica, Low Amplitude Fatigue of Copper Single Crystals-II. Surface Observations, Vol. 33, (1985), pp. 1307-1317.

(3) Ma, B. T. and Larid, C., Acta Metallurgica, Overview of Fatigue Behavior in Copper Single Crystals-I. Surface Morphology and Stage I Crack Initiation Sites for Tests at Constant Strain Amplitude, Vol. 37, (1989), pp. 325-336.

(4) Suresh, S., Fatigue of Materials, Second Ed., Cambridge, (1998), pp. 132.

(5) Polak, J., Cyclic Plasticity and Low Cycle Fatigue Life of Metals, Elsevier, (1991), pp. 123.

(6) Thompson, N., Wadsworth, N. and Louat, N., The Origin of Fatigue Fracture in Copper, Philosophical Magazine, Vol. 1, (1956), pp. 113-126.

(7) Winter, A. T., A Model for the Fatigue of Copper at Low Plastic Strain Amplitudes, Philosophical Magazine, Vol. 30, (1974), pp. 719-738.

(8) Buque, C., Persistent Slip Bands in Cyclically Deformed Nickel Polycrystals, International Journal of Fatigue, Vol. 23, (2001), pp. 459-466.

(9) Hunsche, A. and Neumann, P., Quantitative Measurement of Persistent Slip Band Profiles and Crack Initiation, Acta Metallurgica, Vol. 34, (1986), pp. 207-217.

(10) Polak, J., Vasek, A. and Obrtlik, K., Fatigue Damage in Two Step Loading of 316L Steel I. Evolution of Persistent Slip Bands, Fatigue \& Fracture of Engineering Materials \& Structures, Vol. 19, (1996), pp. 147-155.

(11) Pawlowski, Z., Proc. Vib. Prob. 4 (1963), pp. 43-64.

(12) Truell, R. and Hikata, A., Fatigue and Ultrasonic Attenuation, Symposium on Nondestructive Testing, ASTM STP 213, Pennsylvania, USA, (1957), pp. 63-77.

(13) Hirao, M. and Ogi, H., Resonance EMAT System for Acoustoelastic Stress Evaluation in Sheet Metals, Review of Scientific Instrument, Vol. 64 (1993), pp. 3198-3205.

(14) Hirao, M., Ogi, H., Suzuki, N. and Ohtani, T., Ultrasonic Attenuation Peak during Fatigue of Polycrystalline Copper, Acta Materialia, Vol. 48, Issue 2, (2000), pp. 517-524.

(15) Ohtani, T., Nishiyama, K., Yoshikawa, S., Ogi, H. and Hirao, Ultrasonic Attenuation and Microstructural Evolution throughout Tension-Compression Fatigue of a Low-carbon Steel, Materials Science and Engineering A, Vol. 422, Issue 1-2, (2006), pp. 466-470.

(16) Islam, M.N. and Arai, Y., Ultrasonic Back Reflection Evaluation of Crack Growth from PSBs in Low-cycle Fatigue of Stainless Steel under Constant Load Amplitude, Materials Science and Engineering A, Vol. 520, (2009), pp. 49-55.

(17) 'Stainless steel alloys specification'. JIS G4304, Japan Industrial Standard 2002.

(18) Polak, J., Degallaix, S. and Degallaix, G., The role of cyclic slip localization in fatigue damage of materials, Journal de Physique III, Vol. 3 (1993), pp. 679-684.

(19) Granato, A. and Lücke, K., Theory of Mechanical Damping due to Dislocations, Journal of Applied Physics, Vol. 27, (1956), pp. 583-593.

(20) Wolfenden, A., Internal Friction Study of AISI 410 Stainless Steel, Scripta Metallurgica Vol. 17, Issue 3, (1983), pp. 321-325.

(21) Bailat, C., Groschel, F. and Victoria, M., Deformation Modes of Proton and Neutron Irradiated Stainless Steels, Journal of Nuclear Materials, Vol.276, (2000) pp. 283-288. 
(22) Obrtlik, K., Robertson, C.F. and Marini, B., Dislocation Structures in 16MND5 Pressure Vessel Steel Strained in Uniaxial Tension, Journal of Nuclear Materials, Vol. 342, (2005) pp. 35-41.

(23) Repetto, E. A. and Ortiz, M ., A Micromechanical Model of Cyclic Deformation and Fatigue-Crack Nucleation in f.c.c. Single Crystals, Acta Materialia, Vol. 45, (1997), pp. 2577-2595.

(24) Hatanaka, K., Yamada, T. and Hirose, Y., An Effective Plastic Strain Component for Low- cyclic Fatigue of Metals, Bulletin of JSME, Vol. 23, No. 180, (1980), pp. 791-798. 